\title{
Phylodynamics of pertussis in the vaccine era: transition to re-emergence
}

\author{
Ana I. Bento ${ }^{1,2,3}$, Rahil Taujale ${ }^{4}$, Corrie Schot $^{5}$, Thijs Bosch ${ }^{5}$, Rob Mariman $^{5}$, Aaron A. King ${ }^{6}$ \& Pejman Rohani ${ }^{2,3,7}$
} November 10, 2019

${ }^{1}$ School of Public Health, Department of Epidemiology and Biostatistics, Indiana University, Bloomington, IN, USA.

${ }^{2}$ Odum School of Ecology, University of Georgia, Athens GA USA.

${ }^{3}$ Center for the Ecology of Infectious Diseases, University of Georgia, Athens, GA USA.

${ }^{4}$ Institute of Bioinformatics, University of Georgia, Athens, GA, USA.

${ }^{5}$ Centre for Infectious Disease Control (Cib), National Institute for Public Health and the Environment (RIVM), Utrecht, Netherlands.

${ }^{6}$ Center for the Study of Complex Systems, University of Michigan, Ann Arbor, USA.

${ }^{7}$ Department of Infectious Diseases, University of Georgia, Athens, GA, USA.

\section{Abstract}

Bordetella pertussis causes whooping cough in humans. Despite high routine vaccination coverage, pertussis has become the most prevalent vaccine-preventable disease in many industrialized countries, since the 1990s. While a plethora of candidate explanations for the resurgence remain hotly debated, two prevailing ideas focus on vaccine driven pathogen adaptation and on the switch to the latest generation vaccines. We use a suite of genomic and epidemiological approaches to characterize pertussis transmission in the Netherlands, between 1949 and 2017, investigate drivers of B. pertussis population diversity, and the role of allele shifts in the resurgence. Reconciling the epidemiological data with sequence data has allowed us to characterize a resurgence that is not accompanied with increased transmission but has unique functional genomic signatures of decreased diversity. Here we show a positive association between genetic diversity and incidence which validates the hypothesis that the resurgence is associated with the expansion of more pathogenic strains. We see a direct effect of vaccination on the replicative fitness of pathogens. Our models suggest, large outbreaks are unlikely to be due to immune evasion and the vaccine switch cannot be contributing to increased pertussis incidence. Therefore, efforts aimed at mitigating the resurgence in the population at large, and particularly in vulnerable infants, are more likely to succeed by maintaining routine vaccine coverage high.

\section{Main}

Pertussis is a highly contagious disease, primarily caused by the bacterium Bordetella pertussis (1-5). It was historically considered a childhood disease, with a mean age of infection in the pre-vaccine era between 4-6 years $(1,7)$. Research on vaccine development started more than a century ago (1), due in part to high child mortality and morbidity burden (3). In the pre-vaccine era, there were 115,000 to 270,000 cases of pertussis and 5,000 to 10,000 deaths due to the disease each year, in the US alone (3). The roll-out of routine infant vaccination programs starting in many countries during the 1940s and 1950s was associated with notable declines in pertussis disease burden. Indeed, these success of early vaccination efforts (using the whole cell vaccine, DTwP) in reducing incidence led some to consider pertussis as a potential candidate for eradication $(1-3,6)$. However, over the past two decades, high-profile outbreaks in populations with longstanding immunization and high coverage, pertussis is once again a major public health challenge $(1,2,9,11-$ 13). In 2013, the Global Burden of Disease Study estimated pertussis related mortality in the first year of life to be approximately 56000 deaths (14). A definitive explanation for the re-emergence of pertussis remains elusive $(2,8-10,15-17)$, however, there is a great deal of attention paid to the potential role of vaccines on 
(i) pathogen evolution $(15,19)$, (ii) disease severity and asymptomatic carriage $(17,20)$, and (iii) waning of protective immunity and vaccine failure $(16,21,22)$.

The resurgence of pertussis in the Netherlands is a very interesting case study. It has been suggested that B. pertussis in the Netherlands shows vaccine-driven evolution $(15,19,25,26)$. In the Netherlands, the surveillance of pertussis is based mainly on notifications which were made compulsory in 1976. Routine vaccination was introduced in 1953 with a whole cell vaccine (DTwP) (27, Fig. 1). Many changes in vaccination schedule and formulation occurred between 1953 and 2004 (15, Fig. S1): (i) between 1975 and 1984, there was a temporary reduction of DTwP dosage; (ii) in 1999 an acceleration of the first 3 doses to a 2,3 and 4 month schedule; (iii) in 2001 the introduction of an acellular vaccine (Tdap) booster for 4 year-olds and, finally; (iv) in 2005 the replacement of the DTwP by an acellular vaccine (DTaP) (Fig. 1). Almost a decade prior to the switch to DTaP, pertussis incidence increased suddenly in 1996, and since then, has exhibited regular epidemic cycles (27, Fig. 1).

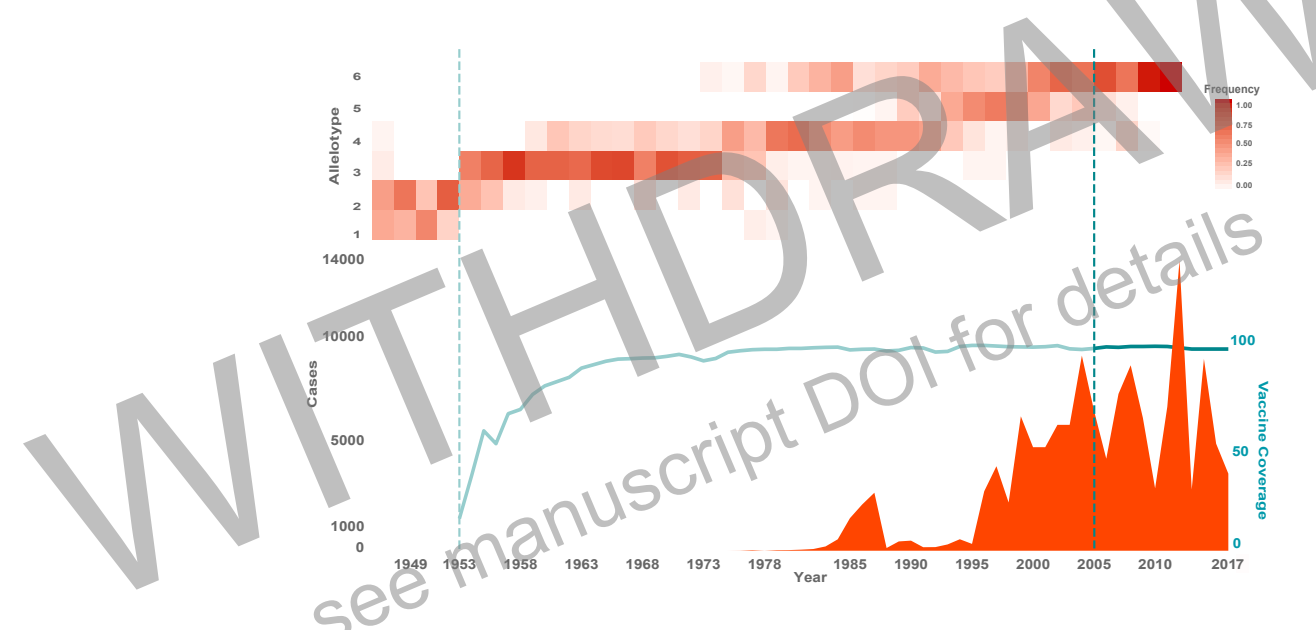

Figure 1: Pertussis in the Netherlands. Temporal trends in allele frequencies of virulence associated $B$. pertussis genes and annual pertussis notifications and vaccine coverage in The Netherlands in the period 1949â2017. Top half of the plot shows $B$. pertussis allelotype frequencies (1949-2017). Allelotypes 1-6 are defined by specific combinations of different alleles of genes related to virulence (Allelotype data obtained from van Gent et al. (25)). Bottom half shows the total number of annual cases in the Netherlands 1976-2017 (red) green line shows vaccine coverage and vertical dotted lines illustrate the introduction of the whole cell vaccine (light green, 1953-2004) and the introduction of the acellular vaccine (2005-Present). NEED TO UPDATE THE TOP PART

Coincident with the resurgence were shifts in B. pertussis allelotype frequencies associated with virulence $(15,19,25,26$, Fig.1). The $B$. pertussis strains circulating during epidemics exhibit diversity visible on a genome structural level that is reflected in allelotype (combinations of difference allelles) frequencies (Fig. 1, top panel). Through time, we observe waves of allelotypes becoming more prevalent and, eventually, going extinct, giving way to new variants (Fig. 1). These allele changes are associated with small mutations, mostly single nucleotide polymorphisms (SNPs), in virulence associated genes - ptxA, ptxP (pertussis toxin), prn (pertactin) and fim3 (fimbriae) $(15,19,25,26)$ (Fig. 2A). Shifts in alleles frequencies, some of which associated with a selective sweeps, provide the most compelling evidence that selective forces are at play $(15,19,25,26)$. Several recent studies have investigated the relationship between the effective population size of viral pathogens and the number of infected hosts (29-31). By harnessing information present in epidemiological time series, genomic (whole genome sequences) and demographic data, we sought to elucidate the relationship between bacterial population diversity and covariates to gain insight into contemporary pertussis epidemiology and evolution. Specifically, we formulated evolutionary models, in an attempt to reconstruct the $B$. pertussis history and transmission dynamics, by integrating parallel data streams of incidence reports (whole population and age-stratified incidence data - Figs. $1 \& 5$ bottom), whole genome isolate sequences, demographic characteristics, vaccine coverage and features, within a coalescentbased framework, that incorporates generalized linear models, for statistical inference. We thus set out to test a set of covariates of evolution and investigate potential drivers of allele shifts and genetic diversity in $B$. pertussis populations, in the Netherlands, and how these shifts might relate to the resurgence of pertussis. Such retrospective knowledge can clarify the past, potentially being of value for rational vaccine design that takes into account the genetic diversity of the bacterium and for predicting the future complexity of regional 
and global B. pertussis genetic diversity.

\section{Results}

\section{Phylogenies}

We used the concatenated SNPs (Tohama I reference, Figs. S2 and S3) to reconstruct the phylogeny. The resulting maximum clade credibility (MCC) time-stamped tree had an estimated root height with a mean of 267.3 years with a 95\% HPD (Highest Posterior Density) interval of [223.5, 317.7] (Figs. S4-S6 - Maximum likelihood estimation).

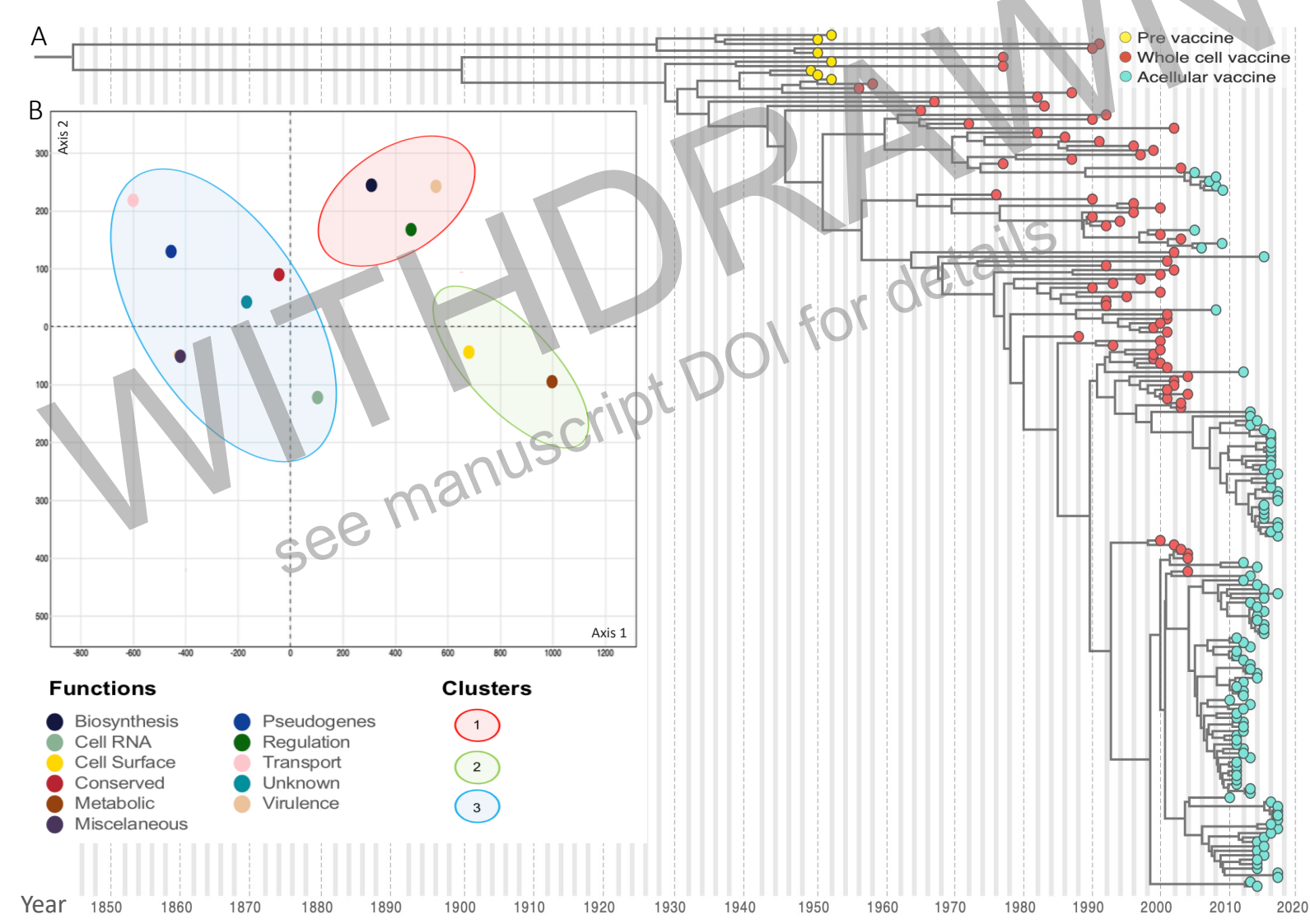

Figure 2: Phylogenetic reconstruction. (A) Maximum clade credibility (MCC) time-stamped tree for Bordetella pertussis in the Netherlands. Estimated and reconstructed with the structured coalescent using Bayesian Markov Chain Monte Carlo methods available in BEAST. Yellow tips (tree) depict pre vaccine era (1949-1952), red depicts whole cell vaccine period (1953-2004) and aquamarine, the acellular-vaccine era (2005-2017). (B) 2D MDS visualization topological tree landscape and clustering of SNP functions. Each dot is color coded matching a function. Shaded eclipses correspond to three separate clusters.

In addition, both MCC (Maximum clade credibility) and maximum likelihood phylogenies estimate root height is estimated between the years 1700 and 1800 (Figs. S4-S6). Further, the reconstructed phylogeny (Fig. 2B) shows a notable change in the population structure of B. pertussis after the introduction of vaccination in the 1950s, exhibiting a ladder-like structure with a long trunk and short side branches. The evolutionary rate of the Dutch population $\left(2.9610^{-4} \mathrm{SNPs} / \mathrm{bp}\right.$ subs/site/year) appears to fall within the expected range for $B$. pertussis previously reported in (24). We find that observed phylogenetic patterns of sequence clustering strongly congruent with vaccine eras. This phylogeny, while representing the whole genome sequence (WGS) of $B$. pertussis, was modelled with the whole genome partitioned into 11 functions. The 24 functional categories defined in Bart et al (24) were further grouped together into 11 broader categories that were used to define the functional categories of the SNPs. These functions depict (i) biosynthesis; (ii) cell process RNA; (iii) cell surface; (iv) conserved regions; (v) metabolism; (vi) miscellaneous; (vii) pseudogenes; 
(viii) regulation; (ix) transport binding; (x) unknown and (xi) virulence (Fig. 3). Partitioning revealed different rates of coalescence, with biosynthesis, virulence, regulation and cell surface functional partitions with the highest rates (respectively $0.679,0.635,0.624,0.615$ ) and the remaining functions with slower rates (the highest being metabolism with 0.56 and the slowest call process RNA functions with 0.467). Similar results are observed when performing this analysis on the SNPs extracted using B1917 (Dutch isolate sequence from 2000).

B. pertussis has been considered a monomorphic pathogen $(2,15)$. Nevertheless, the changes in structure of the population are reflected in the expression diversity and shifts in virulence factors (the set of genes expressed by B. pertussis which allow it to invade and persist inside the host (2)). These proteins expressed by the virulence associated genes are polymorphic $(24,26,32)$. Through time, we observe, in the Netherlands, B. pertussis bacteria circulating during epidemics exhibit diversity visible on a genome structural level that is reflected in changes in allele composition (Fig. 2A) in the pathogen population, with particular alleles becoming dominant and other decreasing in prevalence. As in the phylogenetic reconstruction, we see a decrease in diversity (Figs 2B), also reflected in the entropy of all functions (Figs. S7-S10). PtxA1 allele is detected in the population in the 1980s, quickly going into fixation (Fig. 2A). A similar pattern is observed with ptxP3 which becomes prevalent in the mid 1990s (Fig. 2A), contemporary of the resurgence in 1996 (Fig. 1).

\section{Integrating pertussis epidemiology and molecular evolution}

Different functions show varying coalescent rates thus we expected inferred the demographic history of $B$. pertussis for each of the 11 functions (Fig. 3B) to reflect that. We performed tree topology analysis in which we checked for landscape similarities (33) between trees of the 11 functions of the B. pertussis genome. Kmeans revealed 3 clusters (Fig. 3A): (1) Biosynthesis, Virulence and Regulation SNPs; (2) Metabolism and Cell surface SNPs; (3) all other functions. Number of SNPs differs between functions (Fig. 3B), accounting for selection pressure on each of these functional sub-populations. With varying proportions between $15.6 \%$ (Cell surface SNPs) to 3.5\% (Cell RNA). Similar patterns were observed when using B1917 for SNP extraction (Fig. S5).

We started by estimating $B$. pertussis demography - through effective population size, $N_{e}(39,43,47)$, that characterizes genetic variability - using a Bayesian coalescent non-parametric model with a skygrid prior without testing possible associations of biological significance (39). Reconstructing trends of epidemic growth and decline from pathogen genealogies, allows us to relate pathogen effective population size and time-varying variables that may impact the replicative fitness of a pathogen. We estimated $N_{e}$ using skygrid priors in the model with no covariates (39). Thus, for each of the functions, we estimated demography, treating them as independent sub-populations, as well as for the WGS with 11 partitions (for validation Fig. S6). We observed a similar general trend for all 11 functions (Fig. 3B). Following the introduction of vaccination, in 1953, we observed a slight increase in gene diversity, punctuated by episodes of clonal expansion and retraction (Fig. 3B). In the mid-1990s, there appears to be a marked drop in diversity in most functions apart from the conserved and Cell RNA regions (Fig. 3B). Around 2004, prior to the introduction of routine acellular vaccine, we observed a surge in diversity in virulence and biosynthesis only. Diversity continued to decrease until 2012. In all functions, apart from metabolism, we observed marked diversification in 2012, the year with the highest number of reported cases. After 2012 diversity rapidly dropped until 2017 in most functions. Shannon entropy (Figs. S7-S9) particularly, when following the changes in the ten SNPs with highest entropy through the period 1949-2017) show a similar pattern to our $N_{e}$ estimates. In that we see a reduction in diversity overtime, with some of the functions showing higher standing diversity (i.e. pseudogenes and unknown functional SNPs).

To examine whether we could see a signal of selection, we looked at changes in entropy (Figs. S S7-10) and Tajima's D (49), a statistic used to test the null hypothesis of mutation-drift equilibrium and constant population size, was calculated for all functions separately. A randomly evolving DNA sequence contains mutations with no effect on the fitness and survival of an organism. The randomly evolving mutations are called "neutral", while mutations under selection are "non-neutral". All functions show a negative Tajima's $\mathrm{D}$ value lower than -2 (with a $\mathrm{p}$ value $<0.05$ ), depicting positive populations selection. 


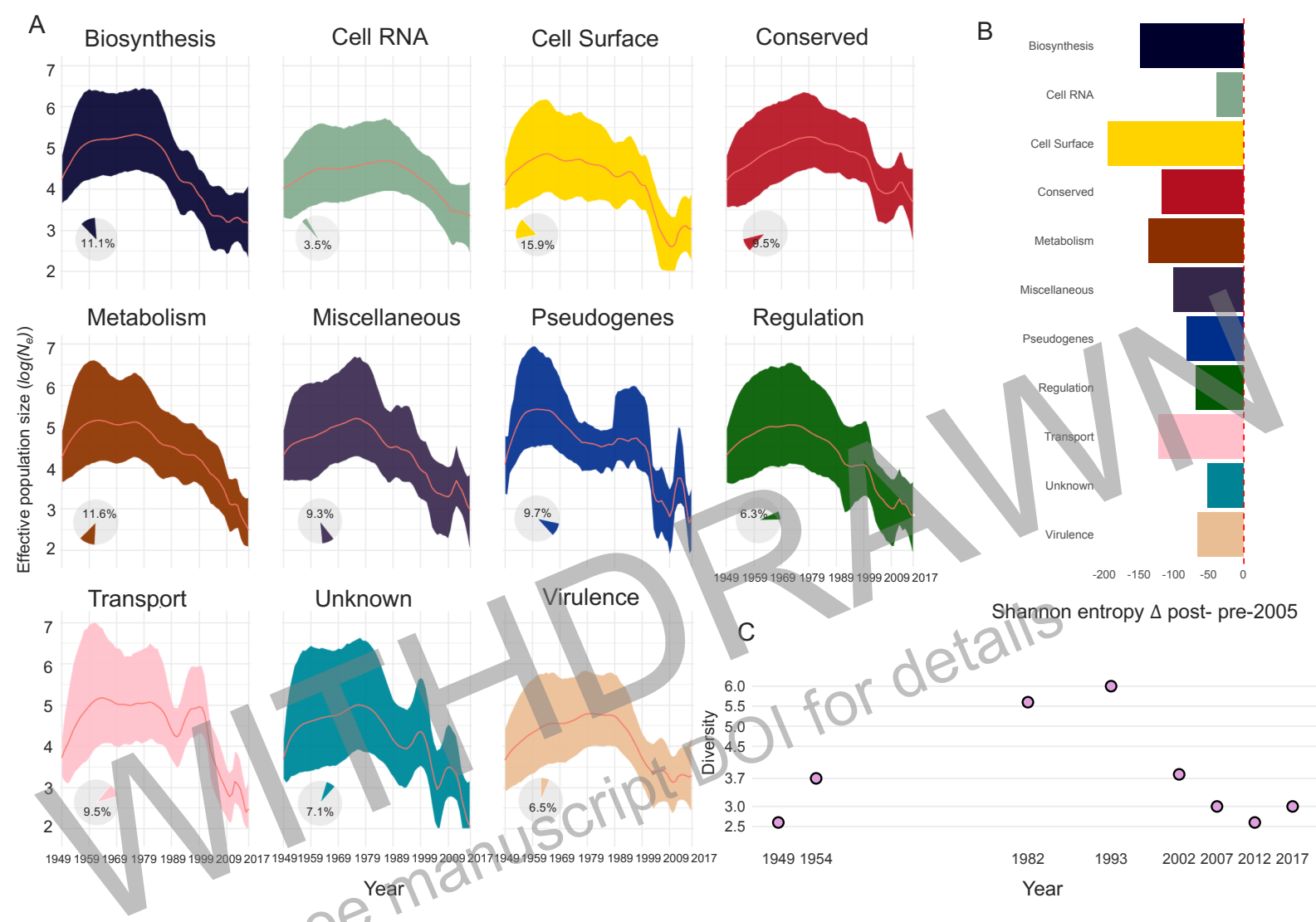

Figure 3: Loss of genomic diversity. (A) Demographic history of B. pertussis in the Netherlands 1949-2017, as inferred via a skygrid coalescent tree priors for each SNP mapped function. Each color matches a function. Shaded interval indicates the $95 \%$ highest posterior density interval of the effective population size, $N_{e}$. Mid-line tracks the inferred mean of $N_{e}$. (B) Shannon entropy differences (data from Fig. S10) between pre- and post- 2005. (C) Effective diversity of dominant virulence factors overtime.

Due to the impact that transmission dynamics and selection can have on pathogen genetic variation (3436 ), we explore associations with potential covariates (annual cases, unvaccinated births and vaccine composition changes) with fluctuations in the effective population size of each of the 11 functions. Thus, we test biological covariates of potential significance alongside estimation of kinetics of gene diversity $N_{e}$ trajectory in time within a generalized linear model (GLM) framework (40). This enables the estimation of the fitness effect of external covariates as well as the prediction of future epidemic dynamics should conditions change (Fig. 4).

The first model explored annual pertussis reported cases as a covariate of $\left(N_{e}\right)$ of each of the 11 functions, to investigate the association of changes in genetic diversity and allele shifts with the resurgence of pertussis (Fig. 4A \& B and Fig. 3). The clusters estimated the 2D multidimensional scaling (MDS) were indicative of the associations observed in the GLMs. Functions in cluster 1 (biosynthesis, virulence and regulation) showed a positive association with reported cases. The coefficients quantifying the effect sizes for the incidence covariate have a posterior mean $\beta_{\text {biosynthesis of } 0.095}(0.008,0.35)$; a posterior mean $\beta_{\text {regulation }}$ of 0.019 $(0.009,0.026)$; a posterior mean $\beta_{\text {virulence }}$ of $0.217(0.003,0.52)$. There is a negative association with the cell surface SNPs and annual reported cases with coefficients quantifying the effect size for the incidence covariate have a posterior mean $\beta_{\text {cell surface }}$ of $-0.06(-0.14,-0.01)$. There was no association between annual reported cases and the remaining 7 functions. 
A
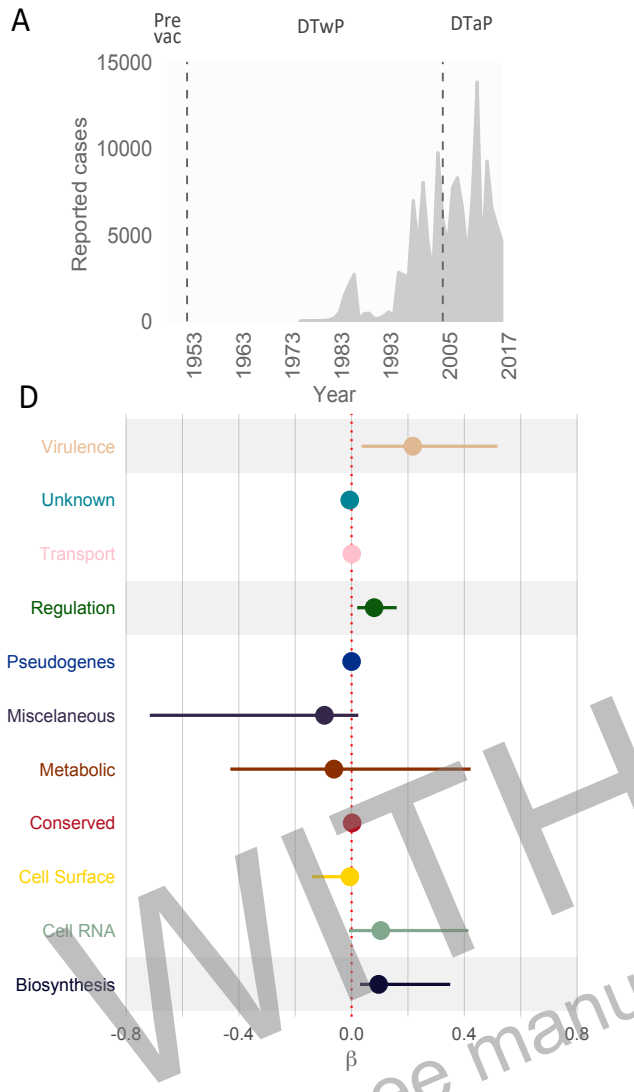

Figure 4: Covariates of demographic history. Covariates explored in the GLMs performed on the demographic history $\left(N_{e}\right)$ of B. pertussis in the Netherlands for the period 1949 till 2017, as inferred via a skygrid coalescent tree prior (Gill et al. 2016). Stationary distributions reached by MCMC with the whole genome (partitioned SNPs into 11 putative functions). (A) Annual reported cases 1976-2017. (B) Coefficient outputs of the GLM testing the association of case reports and genetic diversity. Background dashed lines reflect vaccine epoch: No vaccine, DTwP, DTaP. The vertical dashed line shows 0 . The dots are the coefficients quantifying the effect sizes and the horizontal lines are the 95\% BCI. If BCI includes zero the association is not significant. (C) Annual unvaccinated births 1949-2017. (D) Coefficient outputs of the GLM testing the association of unvaccinated births and genetic diversity. (E) Vaccine changes. No vaccine 1949-1952; DTwP 1953-2004; DTaP 2005-Present. (F) Coefficient outputs of the GLM testing the association of vaccine composition changes and genetic diversity. Solid lines refer to $\beta$ estimates for DTwP and dashed lines refer to $\beta$ estimates for DTaP. The background grey bands highlight the significant associations.

The second model explored annual unvaccinated births as a covariate of $\left(N_{e}\right)$ of each of the 11 functions. We wouldn't expect vaccine coverage to have a significant effect on gene diversity through time, as vaccine coverage quickly rose and remained fairly high. Thus, unvaccinated birth rates, as a proxy for the influx of susceptibles in the population was a more appropriate covariate (Fig. 4C). The only function associated with unvaccinated births is cell surface. The model with $(\log )$ unvaccinated births resulted in a similar trajectory of loss of genetic diversity $\left(N_{e}\right)$ after the introduction of whole cell vaccination with the trajectory punctuated by episodes of clonal expansion, selection and diversification, that track the covariate trajectory (Fig. 4C \& D and Fig 3). The coefficient quantifying the effect size for the unvaccinated births covariate has a posterior mean of $1.4(0.08,3.12)$, indicating a positive significant association.

The final model explored effects of changes in vaccine type as a covariate of $\left(N_{e}\right)$ of each of the 11 functions. This model did not yield associations $N_{e}$ of any of the functions.

From the Shannon entropy analysis, when we investigated in SNPs diversity over the 1949-2017 we see similar trends and patterns of association with annual notifications and susceptible pool (Figs. S7-S10).

Analysis was repeated with B1917 (Figs S12-S17). All associations present with SNPs extracted with Tohama I were validated when using B1917 albeit with slightly different coefficient and $95 \%$ BCI estimates (Fig. S17).

The results of the GLM illustrate several possible dynamics:(i) Increase virulence and increased transmission; (ii) increase virulence decreased transmission; (iii) clonal expansion. 


\section{Resolving with pertussis transmission}

To explore these possibilities, looked at epidemiological data. Since the resurgence in 1996 (Fig. 1) prior to the switch from DTwP to DTaP in 2005, incidence steadily increased, culminating in 2012 with close to 14000 confirmed cases. We picked a Morlet wavelet to deconstruct the annual reported cases time series. The wavelet transform decomposes a signal using functions (wavelets). We recovered dominant periodic components. Incidence remained at a higher level peaking every $3-4$ years (Figs. $1 \& 5$ top). We obtained age-structured pertussis annual case reports for the period 1997-2016 (Fig. 5, bottom). During this period we observed an increasing contribution of the older age groups to the disease burden, particularly individuals in the 10-49 years age groups. This is reflected by the increase in mean age of first infection calculation (pink line, Fig. 5 bottom). Mean age of infection continues to increase, specifically after the introduction of the DTaP in 2005, vaccine mean age of infection oscillates between 20 and 30 years of age.

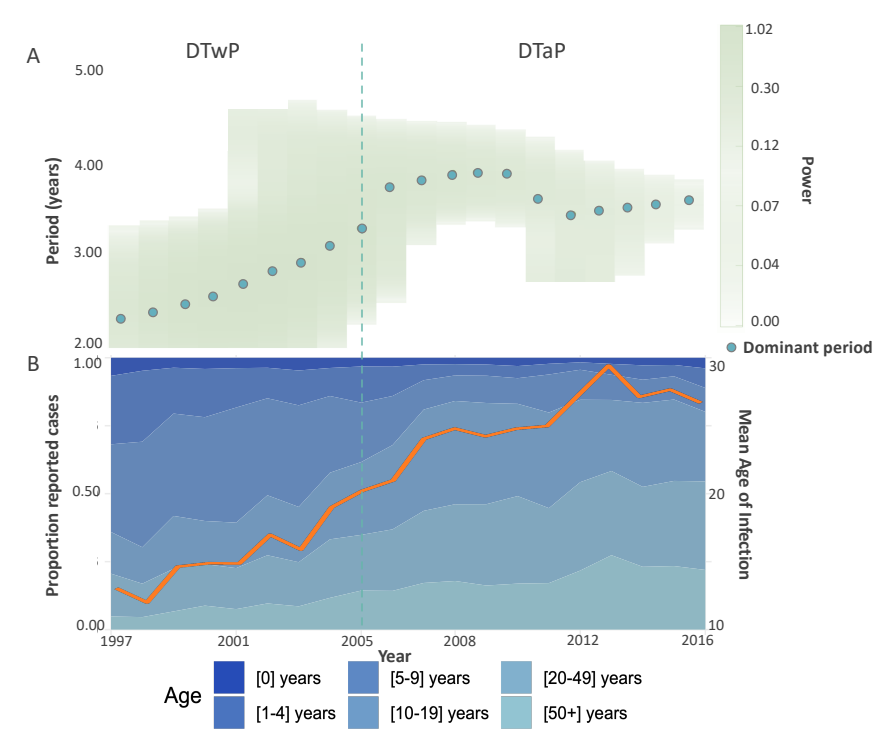

Figure 5: Pertussis transmission dynamics. (Top) Period of the (morlet) wavelet transform of log transformed total annual pertussis cases (dot refer to the dominant period) in the Netherlands for the 1997- 2016 period (Morlet wavelet) with 95\% confidence interval (Shaded green area). Vertical dashed line refers to the year of switch for DTwP to DTaP. (Bottom) Age structure annual pertussis reported cases for the same period. From dark to light individual's age classes increase in age. The orange like depicts increase mean age of infection for the same period.

\section{Discussion}

At first glance, the resurgence and persistence of pertussis despite intense vaccination efforts is unexpected because $B$. pertussis is highly clonal and lacks the genetic diversity of many other pathogens $(2,15,19,37)$. Two potential explanations for the resurgence in the Netherlands are centered on whether recent epidemics are a consequence of waning of vaccine-derived immunity or emergence of novel variants able to escape vaccine protection $(2,8,8,10,10,15,19)$. It is important to distinguish between these explanations, as they warrant distinct public health responses: altering the vaccine schedule versus developing a new vaccine.

Pathogen genomes are a valuable source of information about transmission dynamics (48). Notwithstanding sequences have not been used in conjunction with long-term pertussis epidemiological and demographic data to understand disease dynamics and resurging events. We sought to incorporate these parallel data streams for pertussis in the Netherlands between 1949-2017, by combining the analysis of both epidemiological and phylogeny data with the aim to provide insight on interactions between epidemiological and pathogen evolutionary processes to characterize transmission dynamics to ultimately optimize population specific control strategies.

Many time varying covariates, particularly those with a mechanistic influence on replicative fitness of pathogens, are closely related to the growth rate of epidemic size (48). It is important to assess the re- 
lationship between effective population size and epidemiological dynamics characterizing the number of infections and disease transmission. In this study, we aimed to understand drivers of pertussis transmission in the Netherlands in a period spanning pre vaccination and two different vaccines $(15,19,25)$. Using novel Bayesian coalescent methods that incorporate external potential drivers $(39,40)$, we formally tested hypothesis of vaccine-driven pathogen evolution and resurgence mechanisms. We aimed to examine epidemiologically relevant covariates of genetic diversity, looking for potential genomic signatures (effective population size trends) that may be related with transmission dynamics and that could potentially allow us to elucidate underlying processes driving disease $(29,31,48)$.

The phylogenetic reconstruction revealed a change in structure in the phylogeny (Fig. 2A) which supports previous studies describing strong selection during the vaccine period, suggesting vaccination could act as a major force driving changes in $B$. pertussis populations $(15,19,24-26)$. Polymorphisms in B. pertussis surface proteins have been detected in several countries $(15,19,24-26)$ and variations in pertussis toxin (Ptx) and pertactin (Prn) have been shown to affect vaccine efficacy in a mouse model $(15,19)$. Diversification of gene expression profiles may also influence pathogenicity, which has been suggested in previous studies $(15,19,24-$ 26). Thus understanding selection patterns, could lead to better insight into the mechanisms of pathogen adaptation. While we can harness information from the WGS, trying to infer associations with vaccine and demographic covariates was be more fitting by looking at separate regions of the genome that might be under differential selection pressures. For all putative 11 functions we observed an overall decrease in genetic diversity through time. Decrease in genetic diversity through time can potentially be a snapshot of clonal expansion of those new alleles that appear to have high fitness (15, 19, 24-26) within the population, reduction in transmission (48) as well as sample bias (47). Futher the significant negative Tajima's D (Fig. 3B) potentially depicts selective sweeps (or linkage to a swept gene), and the observed population expansions after a bottleneck $(15,19,24-26)$.

Virulence is the product of complex interactions among evolutionary, ecological and epidemiological processes (53). Thus, it is important to evaluate our results holistically. If taken separately, the phylodynamic GLM analysis on the association between annual reported cases and the putative SNPs for cluster of virulence regulation biosynthesis functions could paint a picture of long-term selection for increase virulence and pathogenicity (Figs $4 \mathrm{~A}-\mathrm{D}$ ). Biosynthesis, cell surface, regulation, metabolic and virulence alleles are regulated similarly in the bvg locus (51). Biosynthesis related genes such as Bordetella liposacharides (LPS) are strongly related with pathogenicity. Virulence genes' products are thought to play a role later in pathogenesis â such as toxins and their secretion systems (52). Mooi and colleagues have also argued that the increased prevalence of ptxP3 in the Netherlands (Fig $2 \mathrm{C}$ ) is associated with increased virulence through production of a more toxic toxin $(15,19,25)$. While the expansion of ptxP3 strains suggests that these strains carry mutations that have increased their fitness $(25,26)$, the assertion that ptxP3 strains produce more pertussis toxin, which results in increased virulence and immune suppression $(25,26)$, is at odds with the epidemiological patterns observed (Figs. $4 \& 5$ ). Further, epidemiological theory (56) illustrates that if transmission was increasing we would see a decrease in age of infection and shorter inter epidemic periods. Annual case reports show a pattern of decreased transmission in the Netherlands (Fig 5). Even though pertussis has resurged in 1996 and incidence remains high, inter-epidemic period and mean age of infection reveal of decrease in transmission (Fig. 5). This pattern, in conjunction with the genomic diversity signatures, highlights the increase in pathogenicity seems to translate in more visible symptoms, rather than infectiousness. This is nonetheless concerning. Thus the much proposed increased virulence or pathogenicity is a product of increased symptomatic cases, offset by increased surveillance. In fact, as transmissability declines we should expect a selection for lower virulence (53).

The positive association between cell surface diversity and unvaccinated births clearly shows the direct effect of vaccine selection pressure on the cell surface alleles.high levels vaccination are expected to lead to a decrease in population diversity, acting as selective pressure whereby only those lineages escaping the vaccine may survive. Indeed, some previous studies have observed such a decrease in population diversity following the introduction of vaccination $(24,54,55)$. The positive association, shows a vaccination effective in maintaining herd immunity through decreasing diversity and reducing prevalence of older lineages. However especially during the DTwP era it seems to fail to completely eradicate older strains, maintaining diversity. As the vaccine coverage decreases observe a rapid increase in diversity. One explanation might be effects on replication cycle which relates with transmission efficiency. Examining the age of patients from which sequences were isolated between 2011 and 2017 (Fig. 6), further validated the hypothesis that rather than 
patterns of immune evasion as previously proposed in the acellular era (post 2005) $(15,19,25)$ what we observe is higher probabilities of cases coming from unvaccinated or individuals who had the DTwP vaccine, perhaps alluding to vaccine failure.

Of the 7 functions not showing direct clear association with epidemiological covariates, it was not surprising that there was no association with metabolic SNPs (Figure 5). The metabolite rich intracellular environment relaxes selective constraints on metabolic genes, which leads to accumulation of mutations, loss of function and gene loss to maximize fitness (53).

Our analysis provided insight into fundamental problems in evolutionary ecology, whilst integrating disparate data streams enhanced our understanding of epidemiological public health issues. Epidemiological and genomic surveillance of populations is crucial understating patterns of pertussis resurgence. The results showed that genomic surveillance, when integrated with epidemiological and demographic data, illustrated a resurgence not driven by immune evasion or enhanced transmission capabilities but resulting in increased pathogenecity whereby there is selection for alleles that appear to be more detectable. A continued integrative approach could prove useful in guiding pertussis control in a resource-efficient manner.

\section{Methods}

\section{Whole genome sequences}

We obtained a total of 193 Whole genome sequences (WGS): downloaded 72 B. pertussis WGS (clinical isolates) with known sampling dates 1949 - 2012 for the Netherlands from publicly available data EMBL/GenBank. 121 whole genome sequences were directly obtained from the Bordetella Unit from the National Institute for Public Health and the Environment (RIVM).

\section{Allelotype and Virulencefactors temporal frequencies}

Allelic variation has been found in virulence-associated genes coding for the pertussis toxin A subunit (ptxA), pertactin (prn), serotype 2 fimbriae (fim2), serotype 3 fimbriae (fim3) and the promoter for pertussis toxin (ptxP). Allelotypes are defined by different combinations of alleles for these genes. Data obtained from van Gent et al. (25) (Fig.1). Virulence factor data (Fig. 2b directly obtained from Bordetella Unit from the National Institute for Public Health and the Environment (RIVM).

\section{Effective diversity $H^{\prime}$}

Measures of how many different types of isolates are present in this population (41). It takes into account both isolate richness as well as the dominance/evenness. If we have two years with equal richness, yet one site is dominated by a single species whereas a second site has a more even abundance of the isolates, then clearly we would consider the second as more diverse. And so the concept of diversity resilience and function.

$$
H^{\prime}=-\sum \frac{n_{i}}{N} \log _{2}\left[\frac{n_{i}}{N}\right]
$$

where $n_{i}$ is the number of individuals of each of the $i$ strains and $N$ is the total number of individuals for the year (or year bins).

\section{Pertussis incidence data}

Annual cases of pertussis were obtained from the National Institute for Public Health and the Environment (RIVM). Publicly avaiable here: https://www.volksgezondheidenzorg.info/onderwerp/ziekten-hetrijksvaccinatieprogramma/cijfers-context/kinkhoest!node-aantal-meldingen-van-kinkhoest

\section{Netherlands demography data}

Official data on age structured population, since 1950 (SI Appendix); population size estimates and birth rate, since 1800, were provided by the Statistics Netherlands (Centraal Bureau voor de Statistiek). 


\section{Pertussis vaccination data}

Vaccine coverage and type data obtained from the National immunization program from the National Institute for Public Health and the Environment (RIVM).

\section{Unvaccinated birth rate}

Unvaccinated birth rate was calculated using annual estimates for population size, birth rate and vaccine coverage.

\section{Single Nucleotide Polymorphisms (SNPs) extraction}

To extract single nucleotide polymorphisms (SNP) from whole genome sequences, we used Tohama I (accession number BX470248) as the reference for alignment $(24,37)$. We used SAMtools (http://samtools.sourceforge.net), in combination with Wgsim to simulate Illumina sequencing for each of the sequences (https://github.com/lh3/wgsim). These reads were then mapped to the reference genome, using BWA-MEM (https://github.com/lh3/bwa), to extract the SNP positions (Fig. S2). SNP positions were controlled for alternate base calls, map, strand and base bias. We validated this procedure using the SNP data available in Bart et al. (24). Additionally, this entire pipeline from the alignment of the reads to the functional annotation of the SNPs was re-run using the B1917 complete genome as the reference (accession number CP009751.1 and assembly version GCA000193595.3) as an alternative measure to cross validate our ebservations and results (Fig. S13). Detailed methods and commands used in this pipeline are provided in the supplementary materials (Appendix SI) to ensure reproducibility.

\section{Functional Mapping}

Each SNP was mapped by additional filtering of SNPs based on functional annotations using Tohama I as reference $(24,37)$. Functional annotations of the identified SNPs were then performed using snpEff v4.3t using these reference genome annotations. The 24 functional categories defined in Bart 2014 were further grouped together into 11 broader categories that were used to define the functional categories of the SNPs. We subsequently used B1917 (2000 isolate) as a reference as validation.

\section{Shannon Entropy}

The shifts in Shannon entropy (42) presented in figure 3 were obtained by first splitting the sequences into pre- and post-2005. Then, for each data set, we calculated the entropy per SNP position:

$$
H(X)=-\sum_{x \in X} p(x) \log _{2} p(x)
$$

where $p(x)$ is the frequency of each SNP variation in our sample. We then calculated the relative change in entropy across SNPs for each gene function:

$$
\Delta H=\sum_{S N P} \frac{H_{\text {post }}-H_{\text {pre }}}{H_{\text {pre }}}
$$

\section{Phylogenetic inference}

Time structured phylogenies were inferred using BEAST 1.8.4 (43). Phylogeny was rooted using Tohama I (37). Molecular evolution was modeled according to a general time reversible (GTR) $+\Gamma_{4}$ substitution model (46) independently across four partitions (codon positions 1, 2, 3 and non-coding intergenic regions). A relaxed molecular clock with branch rates drawn from a lognormal distribution (44) that draws branchspecific rates was used to infer the evolutionary rate from date calibrated tips. All other priors used to infer the phylogenetic tree were left at their default values. All analyses were run for 200 million steps across ten 
independent Markov chains (MCMC runs) and states were sampled every 10000 steps. Complete details are provided in the SI Appendix S5 also on the alternative Phylogenetic inference was repeated using SNPs extracted using B1917 as the genome of reference for validation.

\section{Tajima's D}

Tajima's D (49) is statistic used to test the null hypothesis of mutation-drift equilibrium and constant population size. Tajima's D is computed as the difference between two measures of genetic diversity: the mean number of pairwise differences and the number of segregating sites, each scaled so that they are expected to be the same in a neutrally evolving population of constant size. Tajima's statistic computes a standardized measure of the total number of segregating sites (these are DNA sites that are polymorphic) in the sampled DNA and the average number of mutations between pairs in the sample. The two quantities whose values are compared, are both method of moments estimates of the population genetic parameter $\theta$, and so are expected to equal the same value. If these two numbers only differ by as much as one could reasonably expect by chance, then the null hypothesis of neutrality cannot be rejected. Otherwise, the null hypothesis of neutrality is rejected. This analysis was performed using the R package pegas v0.11.

\section{Phylogenetic trees landscape topology}

Using all 11 functional group trees, we explore landscapes of phylogenetic trees computed as distance matrices between each tree topology. 2D multidimensional scaling (MDS) visualization were generated, as described in Kendall \& Colijn (33). K-means clustering is applied to the distances to choose the number of clusters so as to optimize the Bayesian information Criterion (BIC). ADD MORE DETAILS HERE

\section{Demographic inference}

A flexible non-parametric skygrid tree prior (39) was used to infer B. pertussis Netherlands demography, from molecular data by recovering estimates of effective population size $\left(N_{e}\right)$ - a measure of genetic diversityat 68 evenly spaced grid points across 69 years, ending at the most recent tip in the tree (2017) in BEAST v1.8.4 (43), under a relaxed molecular clock with rates drawn from a lognormal distribution (44) and codon position partitioned (positions $1+2$ and 3$) \mathrm{HKY}+\Gamma_{4}(43,45,46)$ nucleotide substitution models. In addition, a GMRF (Gaussian mean random field) prior is used to smooth the $N_{e}$ trajectories. The overall evolutionary rate was given an uninformative continuous time Markov chain (CTMC). MCMC chains were set up, each running for 200 million states, sampling every 10000 states.

\section{Testing factors associated with $B$. pertussis diversity through time}

The reconstructed phylogeny formed the basis for testing covariates using a generalized linear model (GLM) framework $(39,40)$ that estimates a coefficient of effect size $\beta$ that infers degree of correlation and its significance of each candidate covariate for genetic diversity trajectory through time as the response variable (effective population size - $\left.N_{e}\right)(39,40)$. To determine if a particular covariate can be incorporated in the model, we employed stochastic search variable selection by computing Bayes factors (BFs) for each covariate (45).This approach uses the data to select the explanatory variables and their effect sizes We set the prior for inclusion probabilities $(\delta)$ such that there was a $50 \%$ probability of each covariate being included. For this, $\delta$ is a binary indicator $(0,1)$ variable that allows for calculation of Bayes Factors and thus model selection (45). So at a given interval, $\log \left(N_{e}(t)\right)=\beta \delta \log (\operatorname{cov})_{t}+w_{t}$. The candidate covariates considered were (i) pertussis annual incidence; (ii) unvaccinated births- a proxy measure of both vaccine coverage and demographic changes- and (iii) changes in pertussis routine vaccination namely, vaccine type (whole cell composition changes and its replacement with the acellular vaccine) and in schedule (SI Appendix, Table S4). This methodology is explained in detail here $(39,40)$. Details regarding why most covariates should be $\log$ transformed is in SI Appendix, section S6.1. Details regarding using covariates with missing data are in SI Appendix, section S6.2. Details regarding using covariates as factor levels are in SI Appendix, section S6.3. Details of Bayes factors calculations are in SI Appendix, section S6.4. 


\section{Wavelet analysis}

In order to investigate the periodicity of pertussis epidemics over this period, we used wavelet analysis (50) to decompose time series, to detect the dominant frequencies and dominant period through time. Wavelets are especially powerful when time series data are inherently non-stationary (50). For this analysis, we used the Morlet wavelet of log-transformed total annual cases. This decomposition yields a good localization in both time and frequency, which is well suited for investigating the temporal evolution of aperiodic and transient signals.

\section{Mean Age of Infection}

In the presence of vaccination interventions, mean age of infection $(A)$ is dependent on the effective reproductive number $\left(R_{p}\right)(56)$. We expect mean age of infection to decrease as $R_{p}$ increases. we calculate mean age of infection as:

$$
A=\frac{1}{N} \sum_{i} C_{i} \Delta a_{i}
$$

where, $C_{i}$ is the number of reported cases in each age class, $N$ is the total number of individuals in the population and $\Delta a_{i}$ is the time spent in each age class.

\section{References}

[1] SA Plotkin and WA Orenstein. Vaccines. 3rd edition, WB Saunders, Philadelphia, 1999.

[2] Edited by Pejman Rohani and Samuel V. Scarpino. Pertussis: Epidemiology, Immunology and Evolution. Oxford University Press, Oxford, 2019.

[3] N Wood and P MacIntyre. Pertussis. review of epidemiology, diagnosis, management and prevention. Pediatr Respir Rev, 8:291-212, 2008.

[4] Nicole Guiso. Bordetella pertussis and Pertussis Vaccines. Clinical Infectious Diseases, 49(10):1565-1569, 2009.

[5] Romina Libster and Kathryn M Edwards. Bordetella pertussis and Pertussis Vaccines. Expert review of vaccines, 11:133146, 2012.

[6] NW Preston. The eradication of pertussis by vaccination. The Lancet, 329(8545):1312, 1987

[7] RM Anderson and RM May. Directly transmitted infectious diseases:control by vaccination. Science,215, 1053-1060. 1982

[8] Douglas W Jackson and Pejman Rohani. Perplexities of pertussis: recent global epidemiological trends and their potential causes. Epidemiol Infect, 14:672-84, 2014.

[9] E Miller, C Siegrist and P Tharmaphorpilas. WHO Background paper SAGE. 2014.

[10] Matthieu Domenech de Celles, Felicia MG Magpantay, Aaron A King, and Pejman Rohani. The Pertussis Enigma: Reconciling Epidemiology, Immunology, and Evolution. Proc Roy Soc. B, 283:20152309, 2016.

[11] Maria A Riolo, Aaron A King and Pejman Rohani. Can vaccine legacy explain the British pertussis resurgence? Vaccine, 31(49):5903-8, 2013.

[12] John Drake and Pejman Rohani. The decline and resurgence of pertussis in the US. Epidemics, 3(3-4):183-188, 2011.

[13] Matthieu Domenech de Celles, Felicia MG Magpantay, Aaron A King, and Pejman Rohani. The impact of past vaccination coverage and immunity on pertussis resurgence. Science Translational Medicine, 10(434):eaaj1748, 2018.

[14] T Tan, T Dalby T, K Forsyth, SA Halperin, U Heininger, D Hozbor, S Plotkin, R Ulloa-Gutierrez, CH Wirsing von KÃ Inig. Pertussis Across the Globe: Recent Epidemiologic Trends From 2000 to 2013 Pediatr Infect Dis J., 34(9):e222-32, 2015

[15] Fritz R Mooi, I Van Loo I and A J King. Adaptation of Bordetella pertussis to vaccination: a cause for its reemergence? Emerg Infect Dis, 7:526, 2001.

[16] JD Cherry. The Science and Fiction of the "Resurgence" of Pertussis. Pediatrics, 112(2):405-66, 2003.

[17] JD Cherry. Asymptomatic transmission and the resurgence of Bordetella pertussis. BMC Medicine, 13(1):146, 2015. 
[18] FR Mooi, IHM Van Loo, M Van Gent, Q He, MJ Bart, KJ Heuvelman, SC De Greeff, DI Diavatopoulos, P Teunis, N Nagelkerke, J and Mertsola. Bordetella pertussis strains with increased toxin production associated with pertussis resurgence. Emerg Infect Dis, 15(8):1206-1213, 2009.

[19] FR Mooi, IHM Van Loo, M Van Gent, Q He, MJ Bart, KJ Heuvelman, SC De Greeff, DI Diavatopoulos, P Teunis, N Nagelkerke, J and Mertsola. Bordetella pertussis strains with increased toxin production associated with pertussis resurgence. Emerg Infect Dis, 15(8):1206-1213, 2009.

[20] Christopher Gill, Pejman Rohani and Donald M Thea1 The relationship between mucosal immunity, nasopharyngeal carriage, asymptomatic transmission and the resurgence of Bordetella pertussis. F1000Research 2017, 6(F1000 Faculty Rev):1568. 2017

[21] M van Gent, SC de Greeff, HGJ van der Heide and FR Mooi. An investigation into the cause of the 1983 whooping cough epidemic in the Netherlands. Vaccine, 27(13):1898-1903, 2009.

[22] ED Shapiro. Acellular Vaccines and Resurgence of Pertussis. JAMA, 308(20):2149-2150, 2012.

[23] HE de Melker, JFP Schellekens,SE Neppelenbroek, FR Mooi, HC Rumke, and MAE Conyn-van Spaendonck. Reemergence of Pertussis in the Highly Vaccinated Population of the Netherlands: Observations on Surveillance Data. Emerging Infectious Diseases, 6(4):348â357, 2000.

[24] MJ Bart, SR Harris, A Advani, Y Arakawa, D Bottero, V Bouchez, PK Cassiday, CS Chiang, T Dalby, NK Fry, ME Gaillard, M van Gent, N Guiso, HO Hallander, ET Harvill, Q He, HGJ van der Heide, K Heuvelman, K DF Hozbor, K Kamachi, GI Karataev, R Lan, ALutyska, RP Maharjan, J Mertsola, T Miyamura, S Octavia, A Preston, MA Quail, V Sintchenko, P Stefanelli, ML Tondella, RSW Tsang, Y Xu, SM Yao, S Zhang, J Parkhilland FR Mooi. Global population structure and evolution of Bordetella pertussis and their relationship with vaecination. mBio, 5(2):3e01074-14, 2014.

[25] M van Gent, MJ Bart, GJ Han, SC de Greeff, HGJ van der Heide, J Heuvelman and FR Mooi. Small Mutations in Bordetella pertussis Are Associated with Selective Sweeps. Plos One, 7(9):e46407, 2012.

[26] MJ Bart, HGJ van der Heide, A Zeddeman, K Hermans, M van Gent and FR Mooi. Complete Genome Sequences of 11 Bordetella pertussis Strains Representing the Pandemic ptxP3 Lineage. Genome Announc., 3(6):e01394-15, 2015.

[27] M van Wijhe, SA McDonald, HE de Melker, MJ Postma and J Wallinga. Effect of vaccination programmes on mortality burden among children and young adults in the Netherlands during the 20th century: a historical analysis. Lancet Infect Dis., 16:592â598, 2016.

[28] M van Wijhe, SA McDonald, HE de Melker, MJ Postma and J Wallinga. Estimating the population-level effectiveness of vaccination program in the Netherlands. Epidemiology, 16:592â598, 2017.

[29] David A. Rasmussen, Oliver Ratmann, Katia Koelle. Inference for Nonlinear Epidemiological Models Using Genealogies and Time Series. PLoS Comput Biol 7(8): e1002136, 2011

[30] B Dearlove and DJ Wilson. Coalescent inference for infectious disease: meta-analysis of hepatitis C. Philos Trans R Soc Lond B Biol Sci 368:20120314, 2013

[31] Gytis Dudas, Luiz Max Carvalho, Andrew Rambaut, Trevor Bedford. MERS-CoV spillover at the camel-human interface. eLife 2018;7:e31257, 2018

[32] MJ Bart, M van Gent, HGJ van der Heide, J Boekhorst, P Hermans, J Parkhill, and FR Mooi. Comparative genomics of prevaccination and modern bordetella pertussis strains. BMC Genomic, 11(627):1-10, 2010

[33] Michelle Kendall and Caroline Colijn. Mapping Phylogenetic Trees to Reveal Distinct Patterns of Evolution. Molecular Biology and Evolution, 33(610):2735-2743, 2016

[34] Erik M. Volz, Katia Koell and, Trevor Bedford. Viral Phylodynamics. PLoS Comput Biol 9(3): e1002947, 2013

[35] Simon DW. Frost and Erik M. Volz. Viral phylodynamics and the search for an "effective number of infections". Philos Trans R Soc Lond B Biol Sci., 365(1548): 1879â1890, 2010

[36] Yonatan H Grad and Marc Lipsitch. Epidemiologic data and pathogen genome sequences: a powerful synergy for public health. Genome Biology,15:538, 2014

[37] Parkhill, J and Sebaihia, M and Preston, A and Murphy, LD and Thomson, N and Harris, DE and Holden, MT and Churcher, CM and Bentley, SD and Mungall, KL and Cerdeno-Tarraga, AM and Temple, L and James, K and Harris, $\mathrm{B}$ and Quail, MA and Achtman, M and Atkin, R and Baker, S and Basham, D and Bason, N and Cherevach, I and Chillingworth, T and Collins, M and Cronin, A and Davis, P and Doggett, J and Feltwell, T and Goble, A and Hamlin, N and Hauser, $\mathrm{H}$ and Holroyd, S and Jagels, $\mathrm{K}$ and Leather, S and Moule, S and Norberczak, H and O'Neil, S and Ormond, D and Price, C and Rabbinowitsch, E and Rutter, S and Sanders, M and Saunders, D and Seeger, K and Sharp, S and Simmonds, M and Skelton, J and Squares, R and Squares, S and Stevens, K and Unwin, L and Whitehead, S and Barrell, BG and Maskell, DJ. Comparative analysis of the genome sequences of Bordetella pertussis, Bordetella parapertussis and Bordetella bronchiseptica. Nature Genet., 355:32-40, 2003 
[38] M Suchard, R Weiss and J Sinsheimer. Bayesian selection of continuous-time Markov chain evolutionary models. Molecular Biology and Evolution, 18(3):1001-1013, 2001

[39] MS Gill, P Lemey, SN Bennet, R Biek, B Shapiro and MA Suchard. Improving Bayesian Population Dynamics Inference: A Coalescent-Based Model for Multiple Loci. Molecular Biology and Evolution, 30(3):713-724, 2013

[40] MS Gill, P Lemey, SN Bennet, R Biek, B Shapiro and MA Suchard. Understanding Past Population Dynamics: Bayesian Coalescent-Based Modeling with Covariates. Systematic Biology, 65(6):1041-1056, 2016

[41] Anne Chao and Tsung-Jen Shen Nonparametric estimation of Shannon's index of diversity when there are unseen species in sample Environmental and Ecological Statistics, 10:429-443, 2003.

[42] CE Shannon A Mathematical Theory of Communication The Bell System Technical Journal, 27:379â423, 623â656,1948.

[43] AJ Drummond, MA Suchard, D Xie and A Rambaut. Bayesian phylogenetics with BEAUti and the BEAST 1.7. Molecular Biology and Evolution, 29(4):11969â1973, 2012

[44] AJ Drummond, MA Suchard, SYW Ho, MJ Phillips and A Rambaut. Relaxed phylogenetics and dating with confidence. PLoS Biology, 4:e88, 2006

[45] P Lemey, A Rambaut, AJ Drummond and MA Suchard. Bayesian phylogeography finds its roots. PLoS Comput Biology, 5(9):e1000520, 2006

[46] Z Yang. Maximum likelihood phylogenetic estimaton from DNA sequences with variable rates over sites: Approximate methods. Journal of Molecular Evolution, 39:306-1314, 1994

[47] J Kingman. On the geneology of large populations. J Appl Prob, 19:27-43,1982

[48] EM Volz, SL Kosakovsky Pond, MJ Ward, AJ Leigh Brown, SD Frost. Phylodynamics of infectious disease epidemics. Genetics,183(4):1421-30, 2009

[49] F Tajima. Statistical method for testing the neutral mutation hypothesis by DNA polymorphism. Genetics, 123 (3): 585â95, 1989

[50] I Daubechies. Ten lectures on wavelets. SIAM monographs, Philadelphia, PA:SIAM, 1992

[51] Kyung Moon, Richard P. Bonocora, David D. Kim, Qing Chen, Joseph T. Wade, Scott Stibitz and Deborah M. Hintona. The BvgAS Regulon of Bordetella pertussis. Mbio, 8(5):e01526-17, 2017

[52] Sami AlBitar-Nehme, Soorej M. Basheer, Elisabeth Njamkepo, Jean-Robert Brisson, Nicole Guiso and Martine Caroff. Comparison of lipopolysaccharide structures of Bordetella pertussis clinical isolates from pre- and post-vaccine era. Carbohydrate Research, 378:56â62, 2013

[53] C Toft and SGE Andersson. Evolutionary microbial genomics: insights into bacterial host adaption. Nature Reviews Genetics, 378:56â62, 2010

[54] Litt, DJ and Neal, SE and Fry, NK. Changes in genetic diversity of the Bordetella pertussis population in the United Kingdom between 1920 and 2006 reflect vaccination coverage and emergence of a single dominant clonal type. J. Clin. Microbiol., 47:680â688, 2009

[55] Weber, C and Boursaux-Eude, C and Coralie, G and Caro, V and Guiso, N. Changes in the Dutch Bordetella pertussis population in the first 20 years after the introduction of whole-cell vaccines. Microbiology, 39:4396â4403, 2009

[56] Keeling M, Rohani P. Modeling Infectious Diseases: In Humans and Animals. Princeton University Press, 2007.

Acknowledgements We thank Fritz Mooi and Marjolein van Gent for stimulating conversations regarding pertussis in the Netherlands; Philippe Lemey and Mandev Gill for discussions on demographic inference; Eric Harvill and Bodo Linz for discussions on pertussis gene functions.

Conflict of interests declaration This work was supported by a research grant from the National Institutes of Health (grant number 1R01AI101155) and by MIDAS, National Institute of General Medical Sciences (grant number U54-GM111274).

Competing Interests The authors declare that they have no competing financial interests.

Data and materials availability Raw data, code, and analysis files are available at: https://github.com/anabento/PhyloPertussisNetherlands.

Correspondence Correspondence and requests for materials should be addressed to AIB (email: anisabelbento@gmail.com). 